\title{
Evaluation of physician in triage impact on overcrowding in emergency depart- ment using discrete-event simulation
}

\author{
Qingjin Peng $^{\mathrm{a}^{*}}$, Jie Yang ${ }^{\mathrm{a}}$, Trevor Strome ${ }^{\mathrm{b}}$, Erin Weldon ${ }^{\mathrm{b}}$ and Alecs Chochinov ${ }^{\mathrm{b}}$
}

${ }^{a}$ Department of Mechanical Engineering, University of Manitoba, Winnipeg, MB, Canada

${ }^{b}$ Winnipeg Regional Health Authority Emergency Program and Department of Emergency Medicine, Rady Faculty of Health Sciences, University of Manitoba, MB, Canada

\section{H R O N I C L E}

Article history:

Received: June 132020

Received in revised format: June

242020

Accepted: June 262020

Available online:

August 42020

Keywords:

Emergency Department

Physician Triage

Discrete-event Simulation

Wait Time

Length of Stay

\section{A B S T R A C T}

Emergency department (ED) overcrowding is a common issue in emergency medicine of Canada. Previous studies indicate that adding a physician in triage (PIT) can increase accuracy and efficiency in the initial process of patient evaluations. However, the PIT concept should be thoroughly researched before its widespread implementation can be recommended. This paper introduces the evaluation of impact of PIT on ED patient wait times and length of stay (LOS) using simulation modeling. A discrete-event simulation model of ED is built to simulate and predict the effect of PIT intervention. The model performance is validated using current-state ED flow metrics to quantitatively test multiple alternatives for ED improvements. Results show that the PIT implementation can reduce the ED patient LOS by an average of $34 \%$ and Waiting to be Seen time by $49 \%$ across all scenarios studied. The proposed method can be applied to improve the operation efficiency of healthcare systems in the current pandemic, COVID -19 .

(C) 2020 by the authors; licensee Growing Science, Canada.

\section{Introduction}

Hospital emergency department (ED) provides the medical treatment for patients with a variety of illness. ED is expected to provide timely and effective care. However, different factors in operations affect ED's ability to provide the quality care (Amorim et al., 2019). The growing patient demand and limited medical resources are common challenges in current ED operations across all hospitals (Ordu et al., 2020a; Xu et al., 2019), which results in the patient dissatisfaction and ED pressure (Bahari \& Asadi, 2020). A common term of ED overcrowding is often used when studying ED operations, which refers to a situation where the ED service cannot meet care demands within reasonable time frames (Affleck et al., 2013). ED overcrowding reduces the care quality and increases time of the patient treatment (Amorim et al., 2019; Jilania et al., 2019). ED overcrowding has a critical impact on patients' safety for survival chances of severe patients (Assad and Spiegel, 2020). It also brings challenge to flexible, rapid, and accurate operations, with negative effects for both ED staff and patients (Moustaid and Meijer, 2019). ED overcrowding relates to an excessive number of patients, long patient waiting times and patients Leaving Without Being Seen (LWBS). Consequently, ED overcrowding can result in the rate of medical errors increases and delays in treatments. It can also cause the staff stress, and patient dissatisfaction (Duma and Aringhieri, 2020).

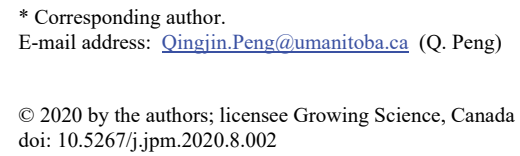


A lot of efforts have been made to reduce ED overcrowding for improving quality of ED operations (Hunter-Zinck et al., 2019). Considering challenges of increasing arrival rates and limited budgets and staff in ED, it is essential to improve the ED efficiency using existing resources (Jilania et al., 2019; Swan et al., 2019). Previous studies indicate that adding a physician in triage (PIT) can increase accuracy and efficiency in the initial process of patient evaluations. Adding one PIT is a redesign process of the patient flow. Normally, nurses conduct triage for patients on their arrivals. In the general protocol of the $\mathrm{ED}$, nurses cannot order any testing for patients in the triage, leaving up to physicians to order necessary testing for patients during the treatment cycle. This may be one of the factors that prolongs the entire treatment cycle, and meantime adds additional workload to physicians. As the PIT concept should be thoroughly researched before its widespread implementation can be recommended, this paper introduces the evaluation of impact of PIT on ED overcrowding based on measures of patient wait times and length of stay (LOS). The research is undertaken at a Hospital in Winnipeg, Canada. The hospital management considers implementing some changes to reduce patient overcrowding in waiting time and throughput time in their emergency care process in order to improve the overall service delivery and system throughput. The ED analysis is challenge as the highly dynamic, complex, and multi-disciplinary nature of ED processes (Duma \& Aringhieri, 2020). ED operates 24/7 including holidays. Therefore, it is impossible to interrupt ED operations system for evaluating their performance (Yousefi a\& Yousefi, 2020). Comparing to the other methods applied in the ED, simulation is much more flexible and versatile to allow free assumptions of the input data such as the arrival pattern and service time, the system structure can be of any complexity, and the custom action logic can be built in to mimic the real system behavior in a great level of details (Kolker, 2008; Duma \& Aringhieri, 2020).

Simulation has been widely applied in the ED improvement. Due to complexity of the ED system, decisions need to be much more evidence-based and made in near-real-time conditions. Simulation model can help analyzing and identifying these types of variability in ED. A computer model can be built to simulate ED operations involving human behavior for complex interaction of ED staff and patients. Different scenarios can be examined in simulation to identify the improvement of ED overcrowding by analyzing the use of different resources and care plans. Therefore, a validated Discrete-event Simulation (DES) model of an ED is built in this research to simulate and predict the effect of PIT intervention. The model's performance is validated using current-state ED flow metrics, and used to quantitatively test multiple design alternatives in ED operations to identify the best-performing staff-schedule configurations. Modeling and analyzing ED systems using simulation has identified that the physician triage generally leads to improved patient length of stay. The decrease in patient wait time to physician diagnosis is likely attributable to the addition of a PIT. Results show that the simulated PIT implementation can reduce the ED patient LOS by an average of 34\% and Waiting to be Seen (WTBS) time by $49 \%$ across all scenarios studied. The optimal scenario reduces LOS by 39\% when a PIT is assigned during 12:000:00 and WTBS by 77\% when a PIT is assigned between 10:00 to 18:00. Following parts of the paper are organised as follows. Next section introduces related research on ED overcrowding and solutions, physician in triage, and ED simulation modeling. ED patient flow and modeling are described in Section 3. Section 4 discusses the PIT strategy for ED improvement. Section 5 introduces scenario experiments and solution search, followed by conclusions and further work of this research in Section 6.

\section{Related research}

\subsection{ED overcrowding}

ED plays a unique role to provide the fast and frontline emergency care, which is a complex operation with uncertainties (McAvoy et al., 2020). ED overcrowding refers to a situation where the demand for emergency services exceeds the ability of an ED to provide the quality care within appropriate time frames (Affleck et al., 2013). ED overcrowding affects the quality and access of health care (Duma and Aringhieri, 2020). ED overcrowding has been a common issue in Emergency Medicine of Canada for many years. The adverse effects of prolonged wait times include the patient dissatisfaction and safety concerns (Bond et al., 2007; Guttmann et al., 2011; Carter et al., 2014). Improving ED operations to alleviate ED overcrowding becomes crucial and urgent (Duma and Aringhieri, 2020; McAvoy et al., 2020; Ordu et al., 2020b). ED is an area with complex interactions of multiple activities in interrelated 
medical processes (Assad and Spiegel, 2020). ED crowding results in long patient lengths of stay. Crowded ED also reduces job satisfaction and productivity. Workload variability, uncertain arrival rates, and resource constraints make it difficult to reduce the ED overcrowding (Swan et al., 2019).

ED overcrowding is also a significant world-wide problem. In Canada, ED overcrowding has been a critical issue in Emergency Medicine for more than 20 years (Affleck et al., 2013). A national level survey conducted by the Canadian Agency for Drugs and Technology in Health reported that $62 \%$ of ED directors regarded overcrowding as a severe problem (Bond et al., 2007), associated with a great risk in patient safety and mortality (Guttmann et al., 2011; Carter et al., 2014). Although factors causing overcrowding penetrate in almost every level of an ED system, the mismatch between the supply and demand devotes its major contribution to this issue (Asplin et al., 2003). There is extensive literature on a variety of potential effects of overcrowding. Previous studies indicate that one of the main factors causing ED overcrowding is inefficiency of the ED process. When the ED demand exceeds hospital resources, ED overcrowding arises to cause increased rate of medical errors (Davis and Fard, 2020). Research also found the negative effect of overcrowding on the patient care and medical staff satisfaction. A comprehensive review concluded that common effects of ED overcrowding include the patient mortality, transport delays, treatment delays, ambulance diversion, patient elopement, and financial effect (Hoot \& Aronsky, 2008; Higginson, 2012). Even though there is not a universal standard to measure the ED crowdedness, an alternative well accepted by the public is to measure the ED crowdedness by monitoring outcome measures, such as patient wait times, patient length of stay, patient throughput, left without being seen, bed occupancy, ambulance diversion rate, and resource utilization (Hoot et al., 2008). A Canadian national survey demonstrated that these outcome measures could be a good projection of the ED crowdedness because their impact on ED overcrowding is obvious (Bond et al., 2007).

To improve the patient flow within an ED, multiple strategies have been proposed, such as ED floor plan modifications, changes in workflows, and resources adjustments (Allihaibi et al., 2020). Multiple solutions to alleviate ED overcrowding have been studied in literature including the demand management in non-urgent patient referrals, ambulance diversion and destination control, additional resources either physical or human, and operations research to provide business intelligence for the patient flow optimization (Hoot and Aronsky, 2008). As it was noted, adding resources is the most direct way to reduce crowding but it is also the most expensive method without the preferred option. Rather than focusing on the level of resources that the ED deserves, it sounds more reasonable to consider how to ensure the ED resources direct to those who need them, the patients in the waiting room (Hopp et al., 2012). There are multiple interventions for potential solutions to improve the ED patient flow (Oredsson et al., 2011; Wiler et al., 2010). Patient care processes can be defined from a patient arrival to the ED to the comprehensive evaluation and treatment of the patient by ED health care providers. Typically, these front-end operations include the initial patient presentation, registration, triage, bed placement, and medical evaluation although they may vary in different EDs as uncertain factors (Wiler et al., 2010). Uncertainty in ED may be unknown arrivals and discharges, unit transfers, and process complications. Variability arises from disease seasonality, holidays, specialized staff, and physical resources (Davis and Fard, 2020). The time spending by completing the front-end operations has influence on the ED total length of stay. Patients would have to wait in a queue because of their non-succession occurring (Wiler et al., 2010). According to Oredsson et al. (2011), these front-end or triage-related interventions can be generally grouped into the team triage, patient streaming, fast track, point-of-care testing, and nurse requested X-ray.

\subsection{Physician in triage (PIT)}

Different strategies of interventions have been proposed to improve ED front-end processing. These strategies include using immediate bedding, bedside registration, tracking systems and whiteboards, wireless communication device, kiosk self-check-in and personal health record technology (Wiler et al., 2010; Kaushal et al., 2015). Some researchers tried to modify triage protocols to smooth the patient flow in Eds (Saghafian et al., 2015). Resource allocation and staffing schedules were also major concerns in some research publications. These different interventions present some potentials to improve the ED performance, but only with methods of the fast track and team triage having relatively strong scientific 
evidence (Oredsson et al., 2011). Fast track is the most studied intervention, the use of a fast tract lane helps to process lower acuity patients more quickly (Kaushal et al., 2015). The advantage of the fast track is significant for patients in non-urgent situations, whereas its effects on urgent patients are unclear (Ardagh et al., 2002). Team triage is designed theoretically for all types of patients regardless of their acuity level. Comparing with the fast track, urgent patients may be better handled by the team triage (Oredsson et al., 2011). Physician in triage (PIT) is one of these strategies to increase accuracy and efficiency in the initial process of patient evaluation by gaining more accurate information upfront, and issuing discharge or appropriate tests early by adding a physician in triage (Oredsson et al., 2011). Theoretically, the PIT method can be beneficial to all types of patients regardless of their acuity levels.

There are a few studies in literature on the use of a physician in triage or team triage for its impact on ED patient flows. Surprisingly, most of these studies come to conclusions that adding a physician in triage improves the overall emergency care services and mitigates effects of ED overcrowding (Subash, 2004; Choi, 2006; Holroyd et al., 2007; Rowe et al., 2011). The traditional "nurse triage" model of managing patient arrivals often creates barriers to the process of efficiently evaluating patients (Shea et al., 2012). By adding a PIT, ED can increase accuracy and efficiency in the initial process of patient evaluations by gaining more accurate information upfront, and issuing discharge or appropriate tests early, meantime patients will benefit from the immediate assessment by a physician (Oredsson et al., 2011). The introduction of a PIT to the team may also enable inexperienced staff to extend their knowledge and practice to a senior level with the mentorship and guidance from the physician (Richardson et al., 2004; Oredsson et al., 2011), thus further advance on efficiency in triage can be expected due to experienced staff. But unfortunately, recent PIT related studies are mainly grounded on either the survey or limited data analysis, such as using the interventional study with retrospective data to evaluate the effect of a PIT on the ED throughput. The scientific evidence of the PIT impact on EDs is far away from enough before its widespread implementation (Rowe et al., 2011). More research is required to examine these preliminary findings using a robust decision tool to determine its applications and limitations (Kaushal et al., 2015).

\subsection{ED simulation modeling}

Simulation models are widely used to test various "what if" scenarios within a minimum amount of time and cost, which has made simulation a widespread tool in the ED improvement (Saghafian et al., 2015; Kolker, 2008). Simulation creates a virtual environment of a real or proposed system to examine its reactions to various conditions, it is favored over analytical solutions when studying complex systems such as ED (Jurishica, 2005). There are a lot of research activities in the ED improvement using simulation. For examples, a discrete-event simulation (DES) model was built to measure a number of key performance metrics of an ED to foresee its activities and form strategic plans (Ordu et al., 2020b). A multimodal simulation method was applied in design thinking throughout the planning and construction stages of a newly renovated ED (Petrosoniak et al., 2020). A streamlining patient flow was simulated as a key dictator for the efficient use of available resources and high quality of care (Alhaider et al., 2020).

A DSE model can address uncertainty related to the surgery duration and length of stay at the hospital (Bovin et al, 2020). The simulation model can test scenarios for the optimal solution. Uncertain arrivals of patients can be represented by discrete scenarios for predicting ED prolonged LOS patients upon triage completion, and investigating changes of ED crowding status and savings of ED bed-hour equivalents to estimates measures of the efficacy of the ED intervention (Ordu et al., 2020b). A series of scenarios were simulated for their combined impact on system performance (McAvoy et al., 2020). The research showed different strategies to lead to different outcomes. Simulated outcomes were also evaluated against past evidence and ED experience. The "What if" scenario analysis was proposed to simulate the ED accessibility with different intervention strategies and system limitations based on the existing resources, accurately identify levels of the most effective staff to improve flow. A DES model was used to evaluate ED waiting time and LOS (McKinley et al., 2020). Outputs show the simulation powerful to evaluate balancing measures. 
For a challenging ED problem in determining the best allocation of medical staff for increasing care demand, a simulation solution was proposed to support ED staff considering uncertain patient arrivals (Marchesi et al., 2020). A simulation model was constructed to investigate different strategies of management and effectiveness of the decision-making process (Shakoor et al., 2020). A simulation model was built to assess potential benefits of expanding the size and opening time of the ED at a UK Hospital to ease ED pressures (Chalk, 2020). DES has been applied in the ED analysis to reduce waiting times, increase productivity and improve delivered services. Key steps of a successful simulation include the formulation of problems, collection of data, and model translation. There are few research publications found in using the simulation model to evaluate the PIT effect on EDs. A team triage method was proposed using a receptionist, a nurse and a physician in triage with reduced patient average throughput time by $26 \%$ based on the simulation (Ruohonen et al., 2006). Although simulation is one of the most widely tools used in decision making for analyzing different processes of highly complicated environments such as the high variability in the patient arrival time and required processes (Shakoor et al., 2020), the simulation application in ED has limitations. It is the ED specific, and not generalizable (Günal et al., 2010; Paul et al., 2010). Substantial diversity exists among such studies in terms of the objective, scope, level of details and calibrations. Rather than a generically available tool, simulation is more of a case-by-case approach (Saghafian et al., 2015).

A physician in triage (PIT) is proposed in this research to improve the ED overcrowding. Solutions are searched using PIT to identify areas of improvement for the ED patient flow based upon a validated simulation model. Using the proposed simulation model to examine the PIT operation, the difference of ED performance is measured. Factors potentially causing overcrowding are detected to understand details that impact ED operations. Various scenarios of the ED improvement are proposed and tested incorporating both resource utilization and process optimizations.

Using the computer simulation, it is proposed to achieve success of modeling the emergency care process and determining the impact of resources on key performance measures such as patient waiting times and length of stay.

\section{ED patient flow and modeling}

\subsection{ED patient flow}

The target hospital provides the healthcare service for community outreach programs, ambulatory care programs and inpatient services through 554 beds and 78 nursing bassinets. The hospital ED is a 24/7 emergency care facility with approximately 42,000 patient visits annually, about 120 patients per day. There is a total of 40 spots (beds/seats) for the patient care divided into six distinct care areas. These are the Resuscitation Room (Resus), Stretcher Bay (SB), ERooms (ERms), Minor Treatment Area (MTA), Reassessment Unit (RU), and Psych Rooms. Patients are assigned to one of these areas (except RU) based on age, acuity level, type of illness after arrival. Fig. 1 shows the ED floor plan. ED patients are divided into five classes of CTAS 1, CTAS 2, CTAS 3, CTAS 4, and CTAS 5 according to Canadian Triage and Acuity Scale (CTAS) National Guidelines. CTAS 1 patients are the most critical patients who need immediate life-saving treatments. CTAS 2 are emergent patients, requiring a rapid medical intervention. CTAS 3 and 4 are assigned to urgent or semi-urgent patients that account for a major percentage of the patients. CTAS 5 are non-urgent patients who may go to clinics instead of the ED owing to their minor symptoms.

A patient visit to the ED usually involves a various series of decisions, activities, and interactions with the ED staff. Patient flows can vary from patient to patient based on the acuity level and diagnosis, and thus it is impossible to classify all ED patient flows exactly. However, a general ED patient flow involves most common decisions, activities, and interactions that a patient will experience. A typical visit to the ED usually involves the pre-bed service, on-bed service and departing the ED. Fig. 2 shows a common process that a patient is assigned to a specific treatment area when entering the ED. The process starts at the patient arrival. Each patient is assigned a CTAS level to characterize the patient type during triage. Fig. 3 describes medical operation procedures for patients after being placed into a designated treatment area. 


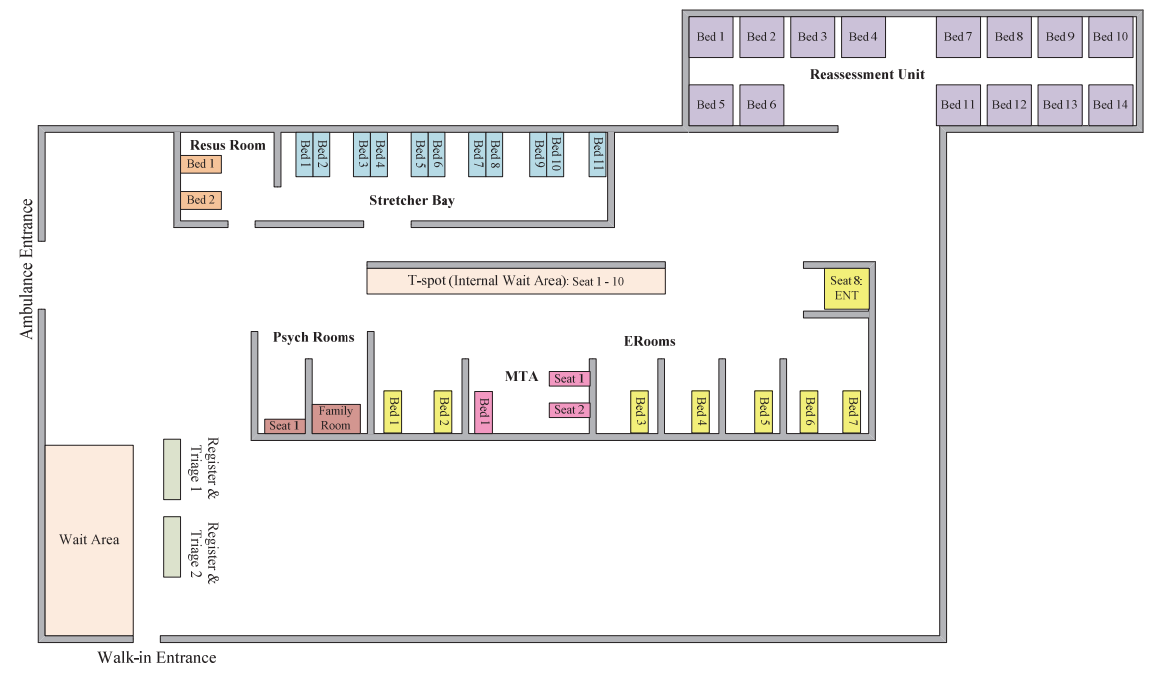

Fig. 1. A simplified ED floor plan

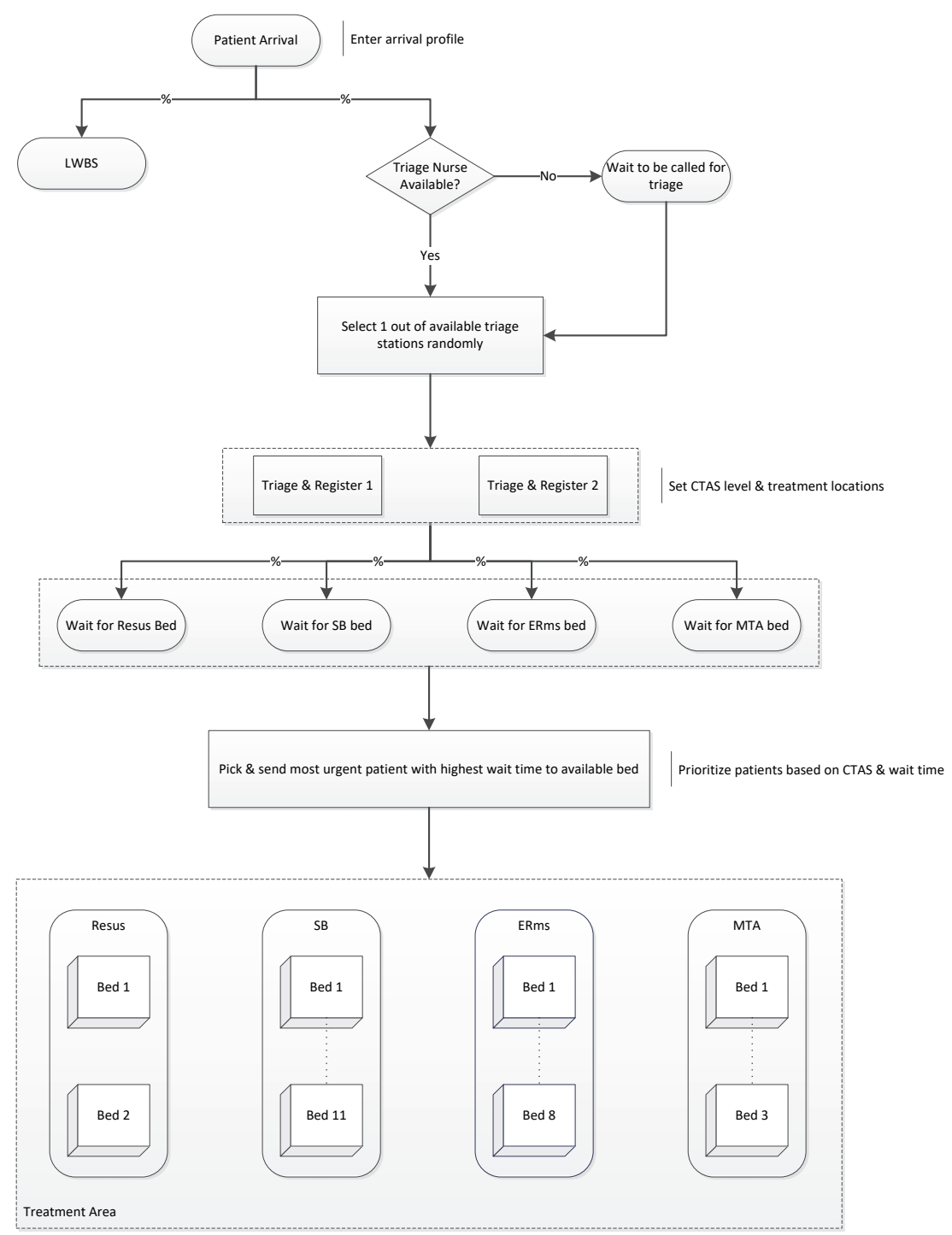

Fig. 2. Treatment area assignment for patient during triage on arrival 


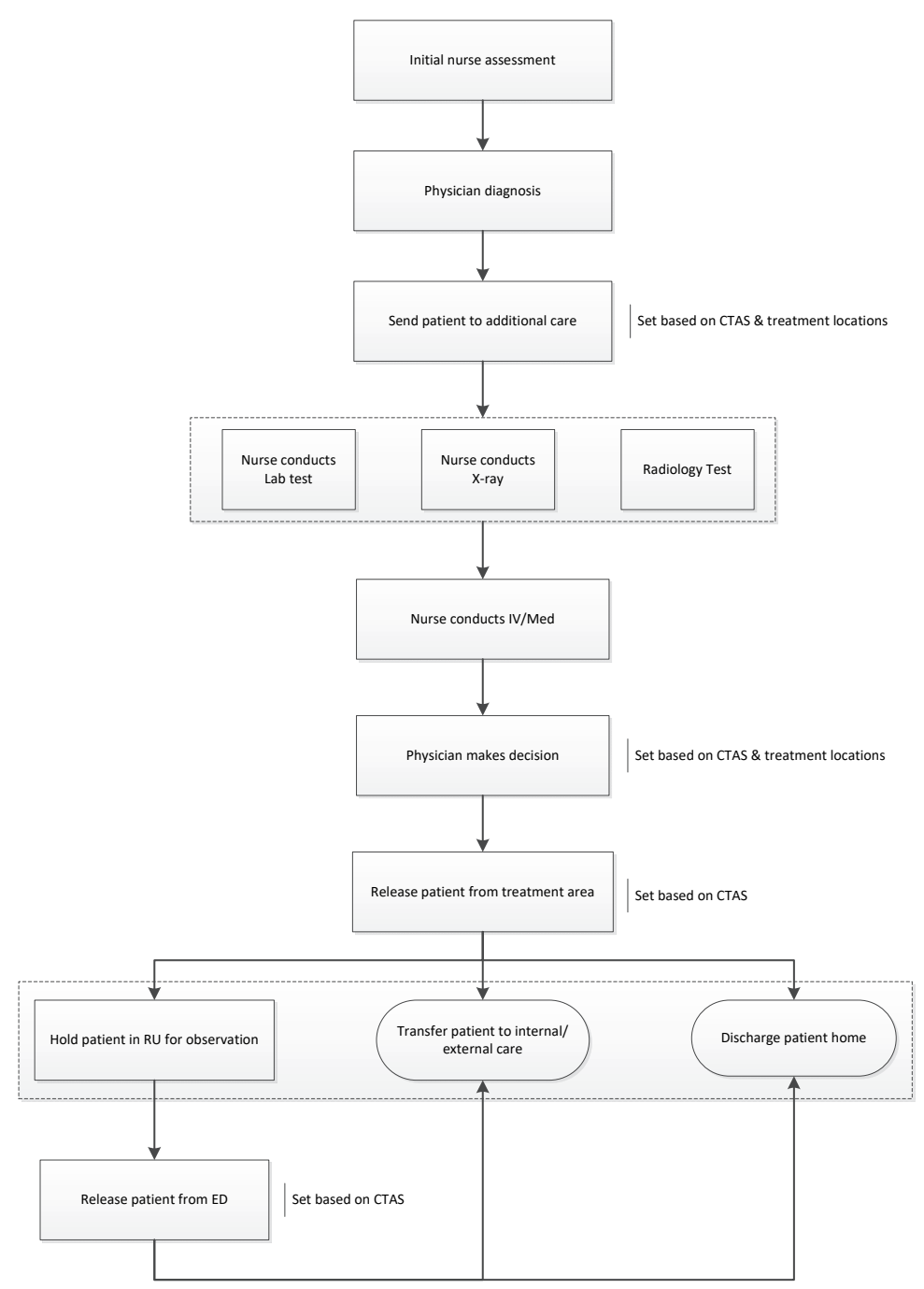

Fig. 3. Medical operation procedures for patients after being assigned into a designated treatment area

\subsection{ED Simulation modeling}

Historical data of one year were gathered from the ED information system provided by the regional health authority with a total of 39,525 anonymous patient records. Each patient record is described in time, location and status stamps in an excel sheet. These data are divided into two sets. The first set consists of data from the first six months and it is used to build the model. The second set contains the remaining six months to validate the model.

The data are grouped based on the nature of contents to model the ED process and then fitted into distributions to drive the simulation. For example, Fig. 4 shows average patient arrivals per hour of a day. Fig. 5 is the occurrence of CTAS 1 to 5 patients in the year.

ED resources are also collected including beds, physicians, nurses, waiting area capacity, current working schedules and treatment area assignments for both physicians and nurses. Operation times of the service procedures are collected from the historical data, and fitted into appropriate distributions.

Patient percentages of discharging home, transferring to other internal or external care services, and holding in RU after patients being seen in one of the treatment areas are also collected for modeling. All these percentages are analyzed based on patient acuity levels for model building considering the ED dynamic environment with numerous rules in patient routing. 
Patient waiting times to see a doctor (i.e. WTBS) and their length of stay in the ED (i.e. LOS) are used as measures to validate the model. To generate credibility of the model, the time spending for a patient from start of triage until the end of active treatment on an assigned room (i.e. WTBS + TIP) is also strictly validated as well as patient daily throughput, total patient arrivals to ED and total patients released from the ED. The simulation model is built using the Witness software system.

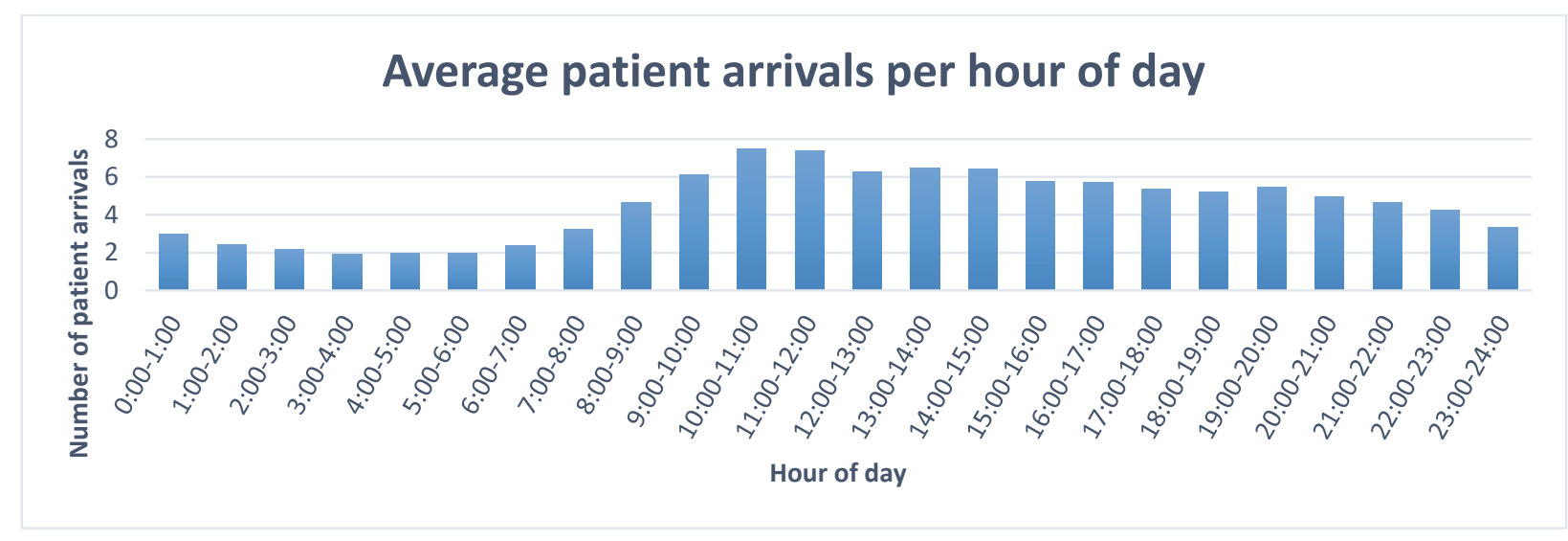

Fig. 4. Average patient arrivals per hour of day

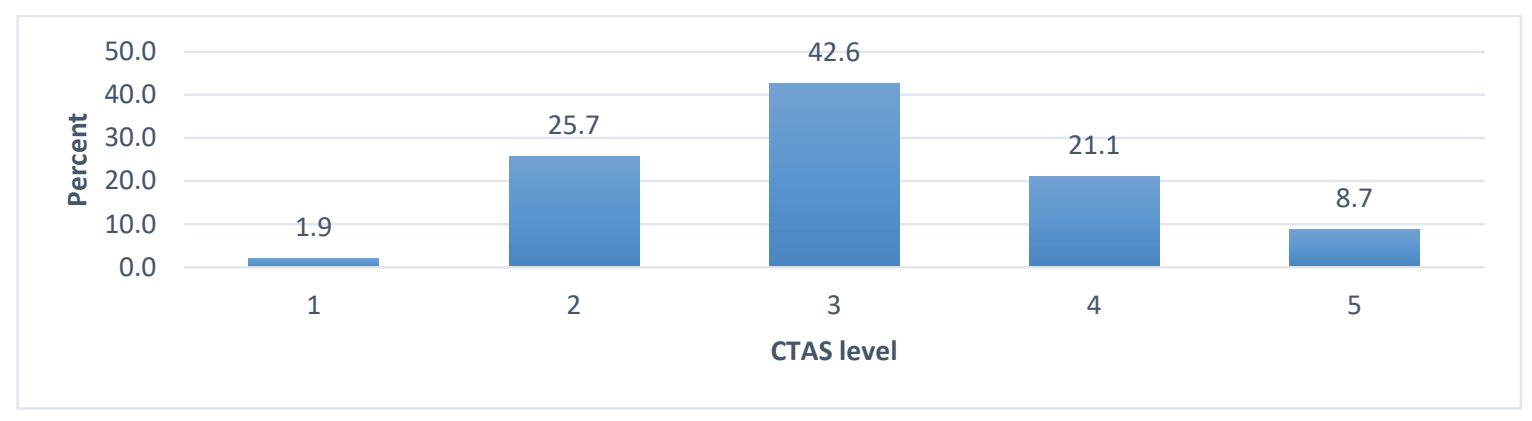

Fig. 5. The occurrence of CTAS 1 - 5 patients in the year

The simulation results with running of 6 months are compared with the system validation data to validate the model. Moreover, hypothesis testing is carried out to see whether there are statistically significant differences in LOS and WTBS for model output data and system validation data as follows.

1) Hypothesis testing for LOS

Null hypothesis $\left(H_{0}\right)$ : There is no statistically significant difference in average LOS for model output data and system validation data.

Alternative hypothesis $\left(H_{1}\right)$ : There is statistically significant difference in average LOS for model output data and system validation data.

2) Hypothesis testing for WTBS

Null hypothesis $\left(H_{0}\right)$ : There is no statistically significant difference in average WTBS for model output data and system validation data.

Alternative hypothesis $\left(H_{1}\right)$ : There is statistically significant difference in average WTBS for model output data and system validation data.

In results of testing, the p-value for LOS hypothesis test is 0.074 while the p-value for WTBS hypothesis test is equal to 0.129 . Since both p-values are greater than the significant level of 0.05 , we cannot 
reject the null hypothesis. In other words, the model is sufficiently validated for the project purpose and can be used for the ED improvement.

\section{PIT Strategy for ED improvement}

The strategy for the ED improvement is to use a physician in triage (PIT), which may increase ED efficiency in the initial process of patient evaluations by gaining more accurate information upfront, and issuing discharge or appropriate tests early. Patients can benefit from the immediate assessment by a physician without waiting for a long time.

For understanding the process using the PIT strategy, a mapping of the patient flow is proposed as shown in Fig. 6. Instead of the current nurse triage model, a team triage based model is built with a physician, a nurse, and a registration clerk in the team. The physician does the initial assessment during triage and orders appropriate tests for patients depending on their needs. The nurse then conducts the tests for patients by following physician's prescription. When patients are waiting for results of testing, the registration clerk quickly collects their basic information for care needs.

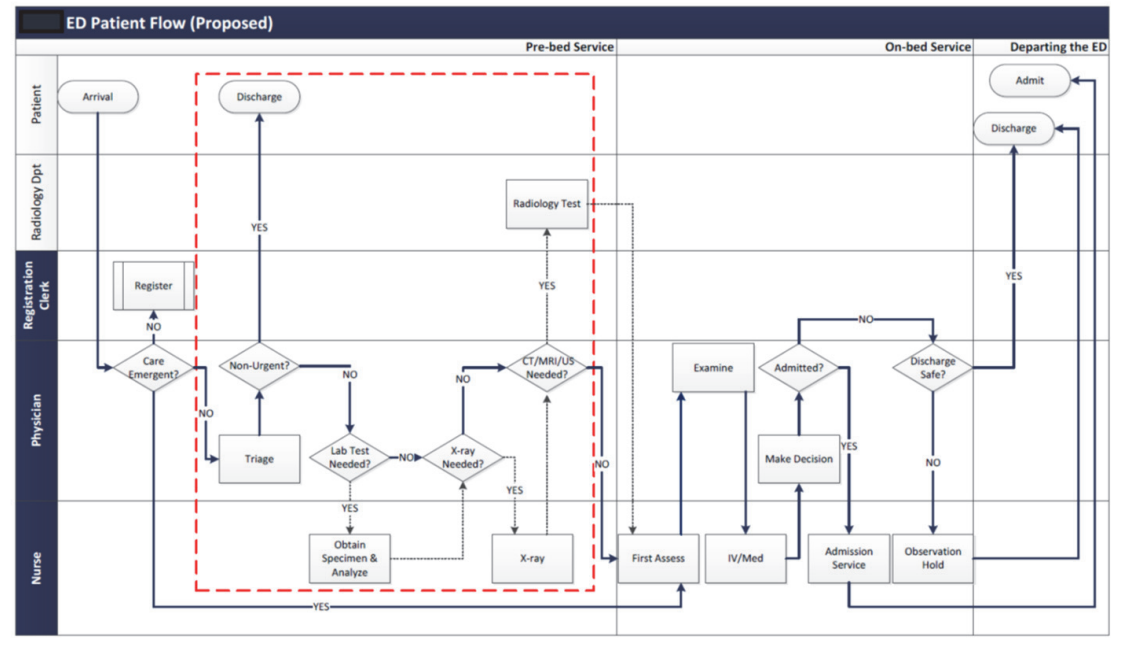

Fig. 6. A proposed patient flow for the ED

In the simulation model, it is assumed that emergent patients who sent to Resus will undertake a second run of testing during the treatment cycle because of their condition complexity. But for patients who are sent to SB, ERms and MTA, they are assumed to take only one run of testing during triage based on their medical needs. In addition, assumptions are made to simplify the modeling effort by eliminating any insignificant parameters and events. The most significant assumptions used in constructing the model are that all patients will remain at the same CTAS level during their stay in the ED, and all patients undergo triage and registration on arrival when the resource is available.

\section{Scenario experiments and solution search}

\subsection{Observing general efficacy of the PIT strategy}

Before jumping to search detailed solutions to implement the PIT strategy, it sounds more rational if we can build some evidence to see its potential efficacy on the ED performance. Therefore, two interventions using the PIT strategy are tested in the simulation model in comparison with the baseline model (Table 1) as follows.

1) Whether there is a need to initiate a triage station with PIT strategy for 24 hours a day (i.e. replacing one nurse triage station which opens for 24 hours a day by a PIT enforced one).

2) What is the level of influence if only one PIT enforced triage station is open to function for 24 hours a day (i.e. cutting off the nurse triage station which only operates during day and evening shift). 
Table 1

Two interventions using the PIT strategy compared to the baseline model

\begin{tabular}{ccccc}
\hline & & PIT Strategy Enforced Triage & \multicolumn{2}{c}{ Current Nurse Triage } \\
\hline \multicolumn{2}{c}{ Shift Hours } & 24 hours a day & 24 hours a day & Day \& Evening Shift \\
\hline Triage & Baseline & 0 & 1 & 1 \\
Station & $P I T-1$ & 1 & 0 & 1 \\
Qty & PIT -2 & 1 & 0 & 0 \\
\hline
\end{tabular}

Note: " 1 " indicates that one triage station is applied, " 0 " denotes non-adoption of the corresponding triage method.

It is important to note that in all three PIT interventions the triage time is reduced to $[1,5]$ minutes for all triage stations. As the introduction of a physician to the triage team may enable inexperienced staff to extend their knowledge and practice to a senior level with mentorship and guidance from the physician, the advance on efficiency in triage can be expected. In the model, the triage time is defined by using a uniform distribution. Since statistical parameters of the triage time by using the PIT strategy are not collectable beforehand, it is assumed that every value in the range of $[1,5]$ minutes is possible.

During this experimentation of using the PIT strategy, we did not consider the option of building more capacity in either triage stations or triage nurses than the current state. Because in the previous experimental test, purely adding more triage stations or triage nurses without changing the current patient flow does not show any great improvement in the patient average waiting time and throughput time. But in order to determine the isolated impact of PIT strategy on the patient flow, the intervention of PIT-2 is tested where only one PIT enforced triage station is open to function for 24 hours a day.

In the PIT testing results, opening only one individual PIT to patients may have an increased risk of stacking patients in the waiting room resulting in even higher numbers in both LOS and WTBS. It is more effective and efficient in the intervention of PIT-1 if we create one PIT enforced triage station and meantime retain one station with nurse triage. Fig. 7 visually displays the difference in LOS and WTBS by using PIT-1 in comparison with the existing measures. PIT-1 may even exhibit greater power of reducing patient average waiting time and throughput time because in the model we do not consider an immediate discharge of patients after triage, but in reality, it may happen quite often if the patient condition is not urgent at all.

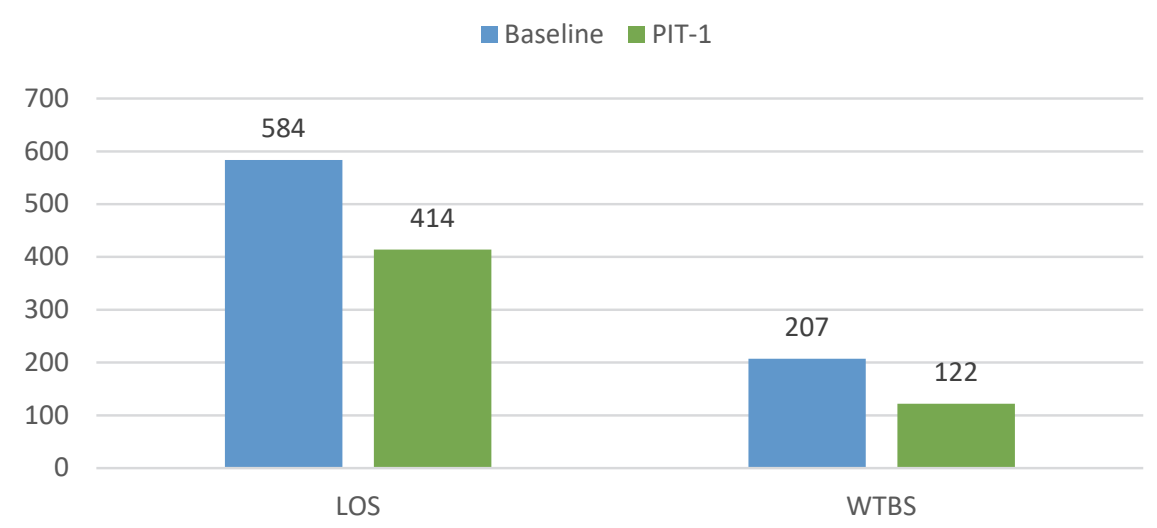

Fig. 7. Results of patient average waiting time and throughput time by using PIT-1 comparing with the baseline (24hrs)

\subsection{Exploring PIT allocation options in busy hours}

According to the previous step of the PIT experimentation, in the case of adding no more nurse resources in triage, the PIT-1 intervention of initiating one triage station with PIT strategy for 24 hours a day and meantime retaining one current nurse triage station for day and evening shifts shows a great positive impact on patient average LOS and WTBS. This step is to break down details in PIT working schedule rather than 24 hours a day to consider how to best allocate a physician in triage within an 8-hour working shift. It is necessary to revisit patient arrival pattern in Fig. 4 in order to meet patient care demands for 


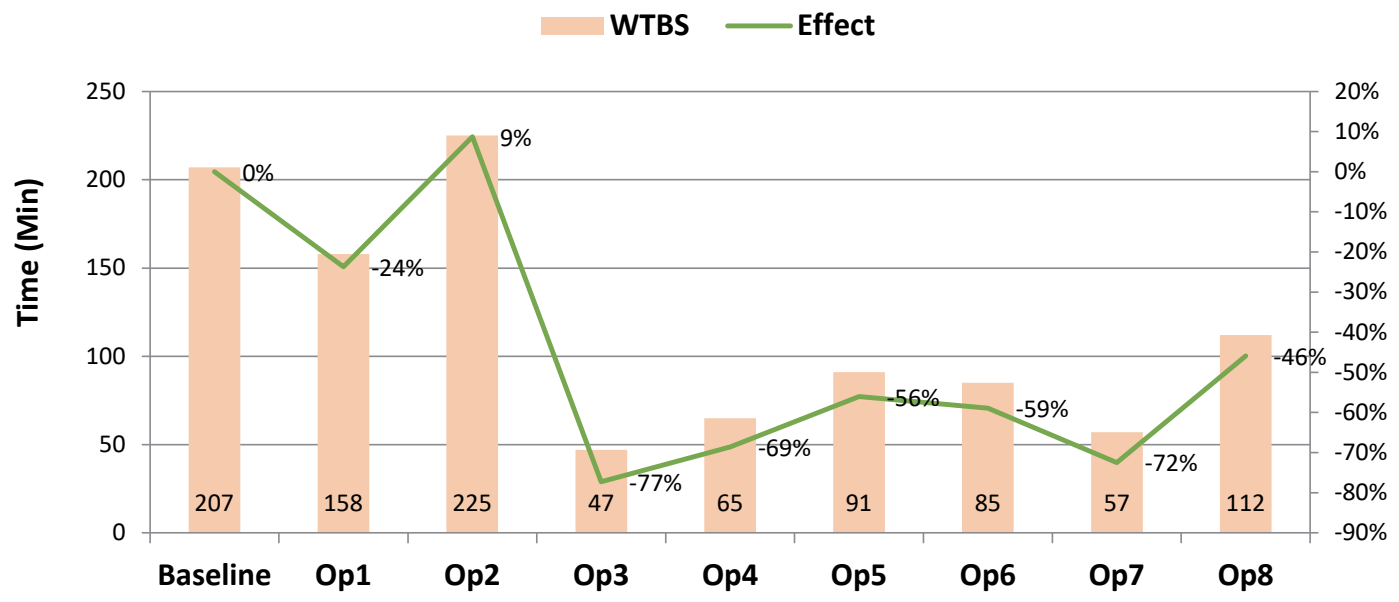

Fig. 9. Results of patient average waiting time by using different PIT allocation options in comparison with the baseline measure ( $8 \mathrm{hrs}$ in busy time)

For changes by using different PIT allocation options, Fig. 8 and Fig. 9 show the comparison of measures of LOS and WTBS with the baseline model performance. Findings are as follows.

1) Fig. 8 shows a great reduction in average LOS by using all eight PIT allocation options, and generally a 34\% reduction in average LOS can be expected by allocating a physician for 8 hours during the busy period of 8:00am - 23:00pm to conduct triage for ED patients.

2) Fig. 9 shows a general great reduction in average WTBS by using PIT allocation options, however, a slight increase in WTBS may be observed.

3) Comparing the reduction in LOS with WTBS, the reduction in LOS fluctuates is less than the reduction in WTBS.

\subsection{Discovering the connection between the PIT allocation and patient care demand}

The PIT experimentation also looks at the influence level on patient average waiting time and throughput time when one physician is allocated in triage during a non-busy period of a day (i.e. 23:00pm-8:00am). Here we name this triage model as PIT-3. The allocation of physician and nurses shares the same rule as the triage model of Op1 except that the physician in PIT-3 is on shift during 23:00pm-8:00am instead of 8:00am - 16:00pm.

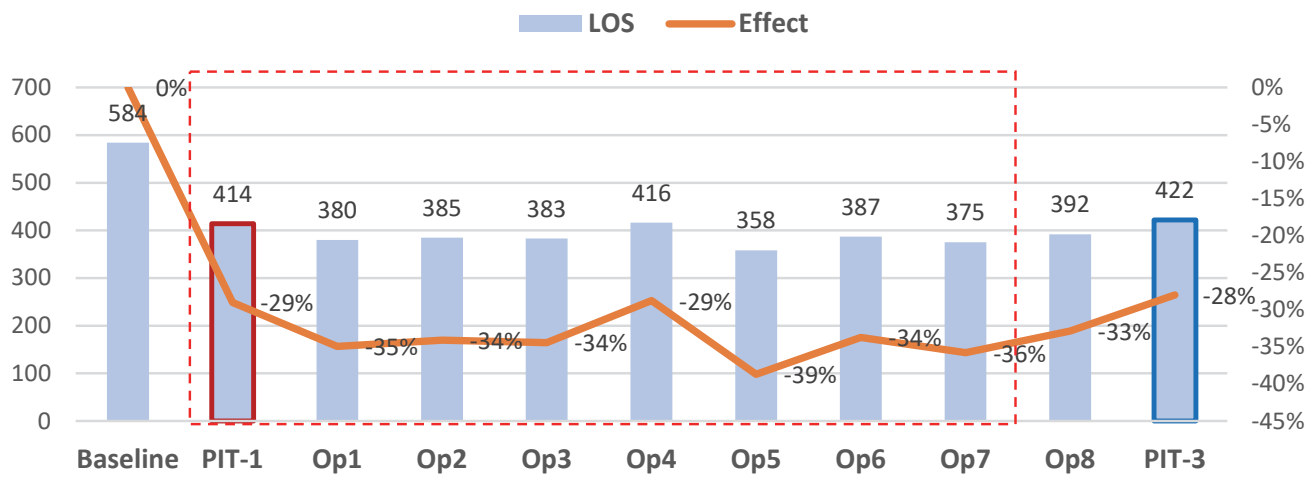

Fig. 10. Effects of allocating a PIT during different hours of a day on patient average throughput time in comparison with the baseline measure ( $24 \mathrm{hrs}$ vs $8 \mathrm{hrs}$ in busy time vs non-busy time)

Figs. 10 and 11 are results of LOS and WTBS by allocating a physician in triage during different hours of a day (i.e. full day [PIT-1] vs. $8 \mathrm{hrs}$ during a busy period of a day [Op1 - Op8] vs. a non-busy period of a day [PIT-3]). By comparison with these two figures, follows can be found. 
1) Applying PIT strategy causes general reduction in average LOS, but its reduction level in LOS does not show a great change in the case of whether a PIT is allocated in busy hours or non-busy hours.

2) In contrast, the allocation of a PIT in non-busy hours greatly increases WTBS in comparison with the baseline measure, its allocation in busy hours tends to fluctuate wild in correspondence to patient care demand for ED service.

3) Recognizing the patient arrival pattern (i.e. care demand) plays an important role in order to determine the best working hours for a PIT so that a greatest reduction in WTBS can be expected. Assigning more working hours for a PIT does not necessarily cause greater reduction in patient average waiting time and throughput time.

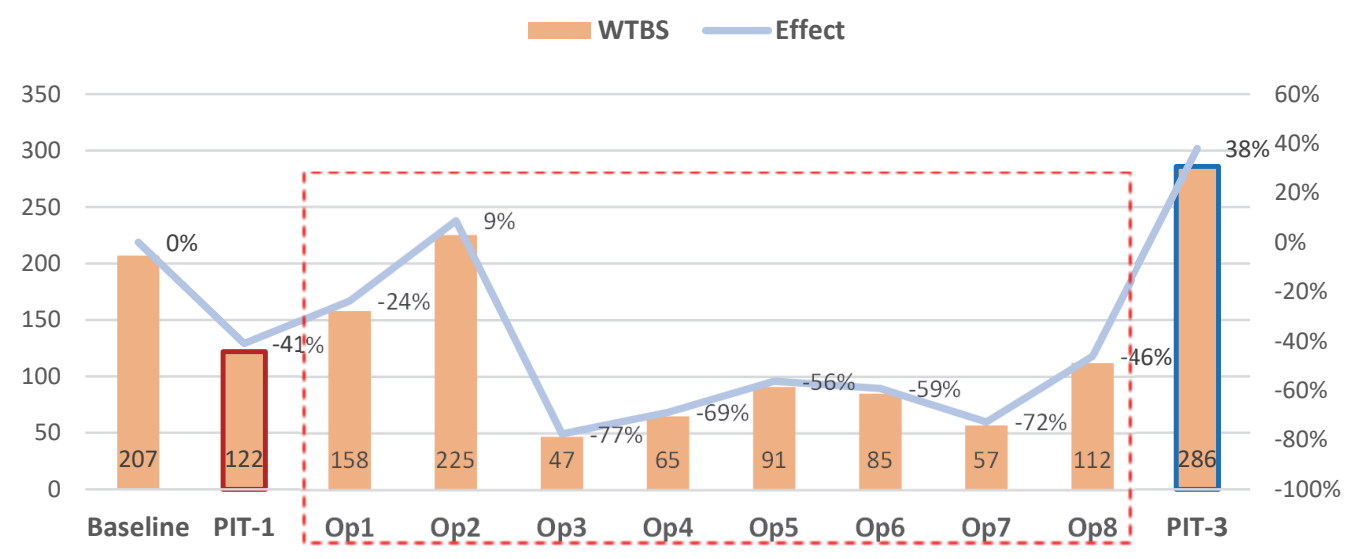

Fig. 11. Effects of allocating a PIT during different hours of a day on patient average waiting time in comparison with the baseline measure ( $24 \mathrm{hrs}$ vs $8 \mathrm{hrs}$ in busy time vs non-busy time)

\subsection{Suggestions for the implementation of PIT strategy}

Based on findings from the PIT experimentation, it is advised that under the current situation of the ED visits assigning a physician during hours of 10:00am - 18:00pm (i.e. Op3) to conduct triage for patients will make the greatest improvement to relieve the overcrowding. The reason of choosing the PIT intervention Op3 is because in Op3 the patient average waiting time to see a doctor is reduced most amongst all the other seven options as shown in Fig. 9, and meantime whether to allocate a PIT in busy hours or non-busy hours does not apparently show a great difference in its reduction level of the patient average throughput time as shown in Fig. 10.

In future, if there are changes to the patient arrival pattern (i.e. care demands or ED visits), the allocation hours of the physician in triage needs to be adjusted correspondingly. The choice when to best allocate a physician in triage is pertinent to the patient care demands for the ED service. A smart decision is to always allocate the physician during the busiest time of the ED to conduct triage for patients.

\section{Conclusions and further work}

Hospital EDs deal with patients with a wide variety of diseases and different urgency, which requires different activities involving human and medical resources (Mahmoudian-Dehkordi \& Sadat, 2020). EDs around the world face challenges of increasing quality services and the limited budget. Enhancing the performance of healthcare services in cost constraints are the prime requirement in healthcare systems (Shakoor et al., 2020). The discrete-event simulation (DES) can imitate processes of ED operations changing over time for improving ED patient flows, optimizing resources, and increasing patients satisfaction.

A validated discrete-event simulation (DES) model was successfully developed in this research to evaluate the existing ED patient flow. By using the validated model, various operation alternatives were tested in order to reduce ED overcrowding and patients waiting time to see doctor and their length of stay in the ED. The patient flow is improved by using the PIT strategy. The validated DES model 
provides a quantitative tool for the analyst to evaluate the influence level on LOS and WTBS among various operating alternatives in comparison with the baseline measures. Contributions are made as follows.

1) Exploring various ED operating alternatives for the hospital management team: Various operating alternatives were explored to improve efficiency of the ED system. As the demand for healthcare continues increasing, the ability to reduce cost, improve efficiency and provide quality service grows in importance. This research has engaged a quantitative approach for hospital decision makers to explore numerous trade-offs between efficiency, workload and capacity.

2) Extending simulation applications in EDs: The use of simulation modeling was demonstrated for predicting the impact of various solutions to the ED overcrowding. Meantime, simulation has shown its great power of examining preliminary findings of a novel solution to determine its application and limitation, practically, for scientific evidence of the PIT impact on EDs.

Further work of this research will search factors that cause ED overcrowding in all levels of the entire healthcare system. The process optimization will also be considered for improving patient flow in the ED and out of the ED. Finding ways to reduce the number of ED visits or to release ED patients fast will also be effective to help alleviating ED overcrowding.

\section{Acknowledgment}

This research is supported by the Research, Education and Innovation (REdI) Fund of the Department of Emergency Medicine, University of Manitoba.

\section{References}

Affleck, A., Parks, P., Drummond, A., Rowe, B. H., \& Ovens, H. J. (2013). Emergency department overcrowding and access block. Canadian Journal of Emergency Medicine, 15(6), 359-370.

Alhaider, A. A., Lau, N., Davenport, P. B., \& Morris, M. K. (2020). Distributed situation awareness: a healthsystem approach to assessing and designing patient flow management. Ergonomics, 63(6), 682-709.

Allihaibi, W. G. M., Cholette, M., Masoud, M., Burke, J., \& Karim, A. (2020). A heuristic approach for scheduling patient treatment in an emergency department based on bed blocking. International Journal of Industrial Engineering Computations, 11, 1-20.

Amorim, F. F., Almeida, K. J. Q. D., Barbalho, S. C. M., Balieiro, V. D. A. T., Machado Neto, A., Dias, G. D. F., ... \& Dasu, S. (2019). Reducing overcrowding in an emergency department: a pilot study. Revista da Associação Médica Brasileira, 65(12), 1476-1481.

Ardagh, M. W., Wells, J. E., Cooper, K., Lyons, R., Patterson, R., \& O'Donovan, P. (2002). Effect of a rapid assessment clinic on the waiting time to be seen by a doctor and the time spent in the department, for patients presenting to an urban emergency department: a controlled prospective trial. The New Zealand Medical Journal (Online), 115(1157).

Asplin, B. R., Magid, D. J., Rhodes, K. V., Solberg, L. I., Lurie, N., \& Camargo Jr, C. A. (2003). A conceptual model of emergency department crowding. Annals of emergency medicine, 42(2), 173-180.

Bahari, A., \& Asadi, F. (2020). A Simulation Optimization Approach for Resource Allocation in an Emergency Department Healthcare Unit. Global Heart, 15(1).

Bond, K., Ospina, M. B., Blitz, S., Afilalo, M., Campbell, S. G., Bullard, M., ... \& Rowe, B. H. (2007). Frequency, determinants and impact of overcrowding in emergency departments in Canada: a national survey. Healthcare quarterly (Toronto, Ont.), 10(4), 32-40.

Bouzon Nagem Assad, D., \& Spiegel, T. (2020). Improving emergency department resource planning: a multiple case study. Health Systems, 9(1), 2-30.

Bovim, T. R., Christiansen, M., Gullhav, A. N., Range, T. M., \& Hellemo, L. (2020). Stochastic master surgery scheduling. European Journal of Operational Research, 285, 695-711.

Carter, E. J., Pouch, S. M., \& Larson, E. L. (2014). The relationship between emergency department crowding and patient outcomes: a systematic review. Journal of Nursing Scholarship, 46(2), 106-115.

Chalk, D. (2020). Using computer simulation to model the expansion needs of the ambulatory emergency care unit at Derriford Hospital. Future Healthcare Journal, 7(1), 60-64. 
Choi, Y. F., Wong, T. W., \& Lau, C. C. (2006). Triage rapid initial assessment by doctor (TRIAD) improves waiting time and processing time of the emergency department. Emergency medicine journal, 23(4), 262265.

Das, D., Pasupathy, K. S., Storlie, C. B., \& Sir, M. Y. (2019). Functional regression-based monitoring of quality of service in hospital emergency departments. IISE Transactions, 51(9), 1012-1024.

Davis, S., \& Fard, N. (2020). Theoretical bounds and approximation of the probability mass function of future hospital bed demand. Health care management science, 23(1), 20-33.

d'Etienne, J. P., Zhou, Y., Kan, C., Shaikh, S., Ho, A. F., Suley, E., ... \& Wang, H. (2020). Two-step predictive model for early detection of emergency department patients with prolonged stay and its management implications. The American Journal of Emergency Medicine.

Duma, D., \& Aringhieri, R. (2020). An ad hoc process mining approach to discover patient paths of an Emergency Department. Flexible Services and Manufacturing Journal, 32(1), 6-34.

Easter, B., Houshiarian, N., Pati, D., \& Wiler, J. L. (2019). Designing efficient emergency departments: Discrete event simulation of internal-waiting areas and split flow sorting. The American Journal of Emergency Medicine, 37(12), 2186-2193.

Greasley, A., \& Edwards, J. S. (2019). Enhancing discrete-event simulation with big data analytics: A review. Journal of the Operational Research Society, 1-21.

Günal, M. M., \& Pidd, M. (2010). Discrete event simulation for performance modelling in health care: a review of the literature. Journal of Simulation, 4(1), 42-51.

Guttmann, A., Schull, M. J., Vermeulen, M. J., \& Stukel, T. A. (2011). Association between waiting times and short term mortality and hospital admission after departure from emergency department: population based cohort study from Ontario, Canada. Bmj, 342, d2983.

Higginson, I. (2012). Emergency department crowding. Emergency medicine journal, 29(6), 437-443.

Holroyd, B. R., Bullard, M. J., Latoszek, K., Gordon, D., Allen, S., Tam, S., ... \& Rowe, B. H. (2007). Impact of a triage liaison physician on emergency department overcrowding and throughput: a randomized controlled trial. Academic Emergency Medicine, 14(8), 702-708.

Hoot, N. R., \& Aronsky, D. (2008). Systematic review of emergency department crowding: causes, effects, and solutions. Annals of emergency medicine, 52(2), 126-136.

Hopp, W. J., Desmond, J. S., Saghafian, S., Van Oyen, M. P., \& Kronick, S. L. (2012). Patient Streaming as a Mechanism for Improving Responsiveness in Emergency Departments. Operations Research, 60(5).

Hunter-Zinck, H. S., Peck, J. S., Strout, T. D., \& Gaehde, S. A. (2019). Predicting emergency department orders with multilabel machine learning techniques and simulating effects on length of stay. Journal of the American Medical Informatics Association, 26(12), 1427-1436.

Jilani, T., Housley, G., Figueredo, G., Tang, P. S., Hatton, J., \& Shaw, D. (2019). Short and Long term predictions of hospital emergency department attendances. International journal of medical informatics, $129,167-174$.

Jurishica, C. J. (2005, December). Emergency department simulations: medicine for building effective models. In Proceedings of the Winter Simulation Conference, 2005. (pp. 7-pp). IEEE.

Kaushal, A., Zhao, Y., Peng, Q., Strome, T., Weldon, E., Zhang, M., \& Chochinov, A. (2015). Evaluation of fast track strategies using agent-based simulation modeling to reduce waiting time in a hospital emergency department. Socio-Economic Planning Sciences, 50, 18-31.

Kolker, A. (2008). Process modeling of emergency department patient flow: Effect of patient length of stay on ED diversion. Journal of Medical Systems, 32(5), 389-401.

Mahmoudian-Dehkordi, A., \& Sadat, S. (2020). A Generic Simulation Model of the Relative Cost-Effectiveness of ICU Versus Step-Down (IMCU) Expansion. Journal of Intensive Care Medicine, 35(2), 191202.

Marchesi, J. F., Hamacher, S., \& Fleck, J. L. (2020). A stochastic programming approach to the physician staffing and scheduling problem. Computers \& Industrial Engineering, 142, 106281.

McAvoy, S., Staib, A., \& Treston, G. (2020). Can a system dynamics model of the emergency department show which levers reduce bottlenecks and delays to improve access to care?. Systems Research and Behavioral Science, 1-19.

McKinley, K. W., Babineau, J., Roskind, C. G., Sonnett, M., \& Doan, Q. (2020). Discrete event simulation modelling to evaluate the impact of a quality improvement initiative on patient flow in a paediatric emergency department. Emergency Medicine Journal, 37(4), 193-199. 
Moustaid, E., \& Meijer, S. (2019, December). A system approach to study waiting times at emergency departments in metropolitan environments. In 2019 Winter Simulation Conference (WSC) (pp. 996-1007). IEEE.

Munavalli, J. R., Rao, S. V., Srinivasan, A., \& van Merode, G. G. (2020). An intelligent real-time scheduler for out-patient clinics: A multi-agent system model. Health Informatics Journal, 1460458220905380.

Ordu, M., Demir, E., Tofallis, C., \& Gunal, M. M. (2020a). A novel healthcare resource allocation decision support tool: A forecasting-simulation-optimization approach. Journal of the Operational Research Society, 1-16.

Ordu, M., Demir, E., \& Tofallis, C. (2020b). A decision support system for demand and capacity modelling of an accident and emergency department. Health Systems, 9(1), 31-56.

Oredsson, S., Jonsson, H., Rognes, J., Lind, L., Göransson, K. E., Ehrenberg, A., ... \& Farrohknia, N. (2011). A systematic review of triage-related interventions to improve patient flow in emergency departments. Scandinavian journal of trauma, resuscitation and emergency medicine, 19(1), 43.

Paul, S. A., Reddy, M. C., \& DeFlitch, C. J. (2010). A systematic review of simulation studies investigating emergency department overcrowding. Simulation, 86(8-9), 559-571.

Petrosoniak, A., Hicks, C., Barratt, L., Gascon, D., Kokoski, C., Campbell, D., ... \& Brydges, R. (2020). Design thinking-informed simulation: an innovative framework to test, evaluate, and modify new clinical infrastructure. Simulation in Healthcare, 15(3), 205-213.

Richardson, J. R., Braitberg, G., \& Yeoh, M. J. (2004). Multidisciplinary assessment at triage: a new way forward. Emergency Medicine, 16(1), 41-46.

Rowe, B. H., Guo, X., Villa-Roel, C., Schull, M., Holroyd, B., Bullard, M., ... \& Innes, G. (2011). The role of triage liaison physicians on mitigating overcrowding in emergency departments: a systematic review. Academic Emergency Medicine, 18(2), 111-120.

Ruohonen, T., Neittaanmaki, P., \& Teittinen, J. (2006, December). Simulation model for improving the operation of the emergency department of special health care. In Proceedings of the 2006 Winter Simulation Conference (pp. 453-458). IEEE.

Saghafian, S., Austin, G., \& Traub, S. J. (2015). Operations research/management contributions to emergency department patient flow optimization: Review and research prospects. IIE Transactions on Healthcare Systems Engineering, 5(2), 101-123.

Shakoor, M., Qureshi, M. R., Jadayil, W. A., Jaber, N., \& Al-Nasra, M. (2020). Application of discrete event simulation for performance evaluation in private healthcare: The case of a radiology department. International Journal of Healthcare Management, 1-8.

Shea, S. S., \& Hoyt, K. S. (2012). "RAPID" team triage: one hospital's approach to patient-centered team triage. Advanced emergency nursing journal, 34(2), 177-189.

Subash, F., Dunn, F., McNicholl, B., \& Marlow, J. (2004). Team triage improves emergency department efficiency. Emergency Medicine Journal, 21(5), 542-544.

Swan, B., Ozaltin, O., Hilburn, S., Gignac, E., \& McCammon, G. (2019, December). Evaluating an emergency department care redesign: a simulation approach. In 2019 Winter Simulation Conference (WSC) (pp. 1137-1147). IEEE.

Wiler, J. L., Gentle, C., Halfpenny, J. M., Heins, A., Mehrotra, A., Mikhail, M. G., \& Fite, D. (2010). Optimizing emergency department front-end operations. Annals of emergency medicine, 55(2), 142-160.

$\mathrm{Wu}, \mathrm{X} ., \mathrm{Xu}, \mathrm{R}$., Li, J., \& Khasawneh, M. T. (2019). A simulation study of bed allocation to reduce blocking probability in emergency departments: A case study in China. Journal of the Operational Research Society, 70(8), 1376-1390.

Yousefi, M., \& Yousefi, M. (2019). Human resource allocation in an emergency department. Kybernetes, 49(3), 779-796.

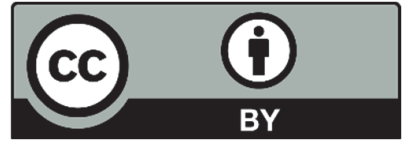

(C) 2020 by the authors; licensee Growing Science, Canada. This is an open access article distributed under the terms and conditions of the Creative Commons Attribution (CCBY) license (http://creativecommons.org/licenses/by/4.0/). 